\title{
The role of localized recoil in the formation of Kikuchi patterns
}

\author{
Aimo Winkelmann ${ }^{\mathrm{a}, *}$, Maarten $\operatorname{Vos}^{\mathrm{b}}$ \\ a Max-Planck-Institut für Mikrostrukturphysik, Weinberg 2, D-06120 Halle, Germany \\ ${ }^{\mathrm{b}}$ Research School of Physics and Engineering, Australian National University, Canberra ACT, Australia
}

\section{A R T I C L E I N F O}

Article history:

Received 3 September 2012

Received in revised form

16 October 2012

Accepted 6 November 2012

Available online 17 November 2012

Keywords:

Kikuchi patterns

Electron backscatter diffraction

Electron channeling patterns

Recoil

Incoherent scattering

Thermal diffuse scattering

\begin{abstract}
A B S T R A C T
In electron scattering from crystals, diffraction spots are replaced by Kikuchi patterns at high momentum transfer. Kikuchi pattern formation is based on the concept of effective incoherent electron sources (or detectors) inside a crystal. The resulting incoherence is a consequence of energy transfer connected with the momentum transfer in large-angle scattering events. We identify atomic recoil as a key incoherent process giving rise to electron Kikuchi patterns in the scope of the "channeling-in and channeling-out" model of electron backscatter diffraction (EBSD) and electron channeling patterns (ECP) in the scanning electron microscope (SEM). Using model calculations, we explore the characteristic role of the localization of the incoherent scattering event at specific places within the unit cell. In this way, we explain why sometimes inelastic losses do cause Kikuchi-type contrast, and sometimes inelastic losses result in the disappearance of this contrast in the SEM.
\end{abstract}

(c) 2012 Elsevier B.V. All rights reserved.

\section{Introduction}

Diffraction methods in the scanning electron microscope (SEM) are valuable tools for the analysis of the microstructure of materials. Depending on the way in which the diffraction information is obtained, we can distinguish two main techniques: electron channeling patterns (ECP) and electron backscatter diffraction (EBSD). EBSD has become a widely applied technique for the analysis of texture, strain and phase analysis [1,2], while the electron channeling technique is especially important in connection with direct imaging of dislocations in the SEM [3-6]. Comprehensive reviews of these methods can be found in [1-4].

Because both techniques are closely linked by the reciprocity principle, the formation process of electron channeling patterns and electron backscatter diffraction patterns can be discussed in a unified way in the context of the "channeling-in and channelingout" model [7-10]. In this context, "channeling" means the result of coherent scattering producing diffraction effects that focus electron waves to different positions within the unit cell. The term "channeling" should not be misunderstood as the classical effect of electron trajectories being somehow confined to the open channels between the atomic planes (as is the case for ion channeling) [11].

The "channeling-in and channeling-out" model puts the atomic nuclei in a key position as they cause the large-angle scattering events required for the backscattered electron signal to be detected. In first

\footnotetext{
* Corresponding author. Tel.: +49 345 5582639; fax: +49 3455511223.

E-mail address: winkelm@mpi-halle.mpg.de (A. Winkelmann).
}

approximation the probability of a large-angle deflection is given by the Rutherford cross section which is proportional $Z^{2}$, with $Z$ the atomic number. The scattering probability can be modulated on the one hand by coherent scattering processes of the incident electron wave prior to the backscattering event. The coherent scattering and interference of the incident plane wave beam is setting up a wave field inside the crystal which can be described as a superposition of Bloch waves. This wave field, depending on the incidence angle with respect to a lattice plane, has a different overlap with the atomic nuclei which translates directly into the modulation of the total backscattering signal from the respective nucleus. By reciprocity, the same types of wave fields are describing the angular modulation of the exit probability of these backscattered electrons as a function of the final direction, i.e. the detection directions ending up on the phosphor screen. Thus coherent scattering of the backscattered electron wave after the backscattering event will also result in a redistribution of the angular intensity. If the backscattered signal is averaged over the exit direction in this unified "channeling-in and channeling-out" model, and we change the incoming direction then we obtain the method of ECP. If we keep the incident beam direction fixed, and study the variations in the outgoing intensity then we are looking at the method of EBSD [8].

It has been shown that the Kikuchi patterns seen in ECP and EBSD can be simulated [12-14,6] using the Bloch wave approach of dynamical electron diffraction well-known from transmission electron microscopy $[15,16]$. These simulations assume that the observed signal is proportional to the intensity of the wave field that is set up by a plane wave incident beam measured at the atomic positions. By reciprocity, the incident plane wave in ECP 
corresponds to an exit wave towards a specific point on the phosphor screen in EBSD.

In order to separate the coherent scattering in the incident part from the coherent scattering in the outgoing part of the total process, the backscattering event needs to break the coherence between these two parts. This means that the phase relationship between the incident wave and the exit wave has to be randomized by the backscattering process. It is instructive to realize that without the incoherent event, we would be simply looking at electron diffraction from a crystal which is described by collective coherent scattering from the crystal atoms. This would result in the conventional spot patterns of transmission high energy electron diffraction (THEED), low energy electron diffraction (LEED), and reflection high energy diffraction (RHEED). These, essentially coherent, techniques are, of course, always additionally influenced by incoherent scattering, which becomes especially important for a quantitative description of transmission electron microscopy measurements in the presence of phonon scattering $[17,18]$. This type of scattering is closely linked to the Kikuchi patterns which are observed in ECP and EBSD.

The nature of the virtual incoherent source inside the crystal which produces Kikuchi patterns is often discussed in rather general terms as caused by "inelastic scattering" or "diffuse scattering". One aim of this paper is to discuss recently obtained clear-cut experimental evidence for the pivotal role of recoil in the formation of Kikuchi patterns. Large-angle scattering of high energy electrons involves significant momentum transfer $\boldsymbol{q}$ (and thus an energy transfer $q^{2} / 2 M_{a}$ with $M_{a}$ the atomic mass) from the incident electron to the incoherently backscattering atom. Experimentally, this recoil energy can be used in a compound crystal to determine the scattering atom. Thus site-specific diffraction information is obtained from the quasi-elastic electrons [19] and this observation suggests new ways to use diffraction for crystallographic analysis in the SEM.

The purpose of this paper is to show (a) the implications of the necessary momentum transfer from the incident electron to the nucleus in the backscattering process, (b) the role of the specific localization of incoherent scattering at different positions inside the crystal unit cell and (c) that an increasing randomization of the incoherent source position over the whole volume of the unit cell leads to the suppression of diffraction information because, effectively, many different possible Kikuchi patterns are averaged in the resulting pattern which is measured. Thus we obtain an understanding when energy losses result in Kikuchi pattern formation, and when it washes them out. This understanding is important as a description of the specific suppression of diffraction information by inelastic scattering in an otherwise perfect crystal is necessary for an improved quantitative simulation of experimental Kikuchi patterns.

The suppression of diffraction information by inelastic scattering was experimentally established in the measurement of the contrast in Kikuchi patterns as a function of the energy loss [20,21]. A clear reduction in diffraction contrast was found when the outgoing trajectory was longer than the inelastic mean free path [21]. Thus a Kikuchi pattern obtained without energy discrimination (e.g. when a phosphor screen is used) contains a large background of inelastically scattered electrons without any diffraction features.

\section{Results}

\subsection{Quasi-elastic backscattering}

In order to demonstrate the decisive role of recoil in Kikuchi pattern formation of the quasi-elastically scattered electrons, we show in Fig. 1 results of angle-dependent electron spectroscopic measurements near zero energy loss from a sapphire sample with

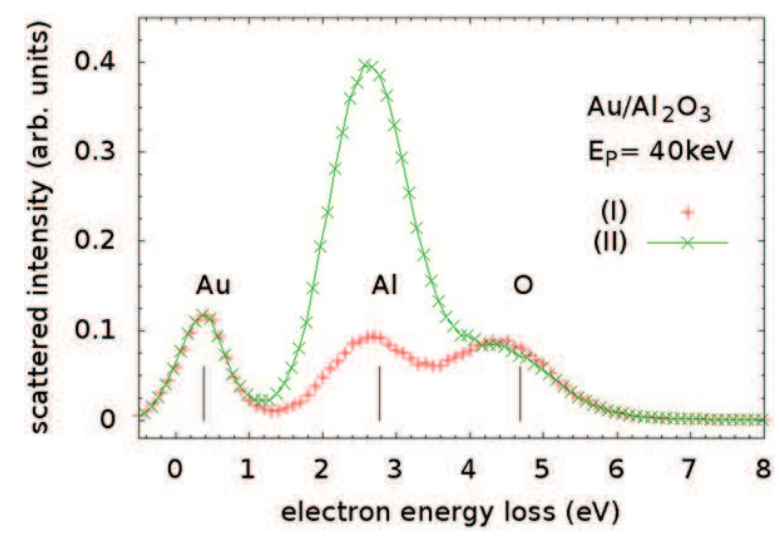

Fig. 1. Energy spectrum of quasi-elastically backscattered electrons from a sapphire $\left(\mathrm{Al}_{2} \mathrm{O}_{3}\right)$ sample with gold atoms deposited on the surface. The vertical lines indicate the recoil loss expected for elastic electron-atom scattering. Curves (I) and (II) are measurements in two different directions. The difference between (I) and (II) is due to diffraction effects.

a small number of $\mathrm{Au}$ atoms deposited on the surface (Au coverage well below a monolayer). These experimental results have been obtained using high resolution electron spectroscopy and analyzed with dynamical Bloch wave simulations as described in detail in $[19,21,22]$.

As can be seen in the data in Fig. 1, the backscattered electron energy distribution shows three peaks which can be assigned to scattering by $\mathrm{Au}, \mathrm{Al}$ and $\mathrm{O}$, respectively. The vertical lines show the expected electron energy loss of $q^{2} / 2 M_{a}$, i.e. what is expected for a classical, billiard-ball type, collision. The agreement of the classical expectations and the observed peak positions is very good, the remaining differences can be assigned to charging of the sapphire sample which results in a reduced scattering energy of about $35 \mathrm{keV}$ [19].

In a quantum-mechanical picture, the recoiling atomic nucleus can be described by a superposition of many simultaneously excited phonons which take up the necessary recoil energy [23]. As a recoil energy of $\approx 1 \mathrm{eV}$ (typical for backscattering from light elements at SEM energies) can excite many, nearly energydegenerate, combinations of phonons, the exact phase relationship between the phonons and the electron is not tractable in each scattering event and the electron wave is thus incoherently scattered by each individual recoiling atom. The time evolution of these phonons results in oscillations spreading from the initially displaced atom over the whole crystal, corresponding to thermal dissipation of the recoil energy. For comparison, the completely coherent reflection of the incident electron by the crystal lattice would result in a single peak at the primary beam energy, since the recoiling mass is macroscopic and the recoil energy loss is infinitesimal. In this case, we would have no information on the mass of any individual atom involved in scattering. The probability of this elastic coherent scattering is described by the Debye-Waller factor, which exponentially decreases with the square of the momentum transfer. Thus, due to the large momentum transfer in the backscattering geometry, elastic coherent backscattering is largely suppressed in the typical EBSD geometry.

In combination with the recoil loss, we simultaneously investigated the influence of diffraction effects. These are seen in the two experimental curves (I,II) presented in Fig. 1 which are recorded for two different exit directions and a fixed incident beam. While Al shows an increase in intensity from (I) to (II), the $\mathrm{O}$ intensity is showing the opposite trend (taking into account that the $\mathrm{O}$ peak is on the wing of the $\mathrm{Al}$ peak). The Au atoms show the same intensity in both cases, which is consistent with the 

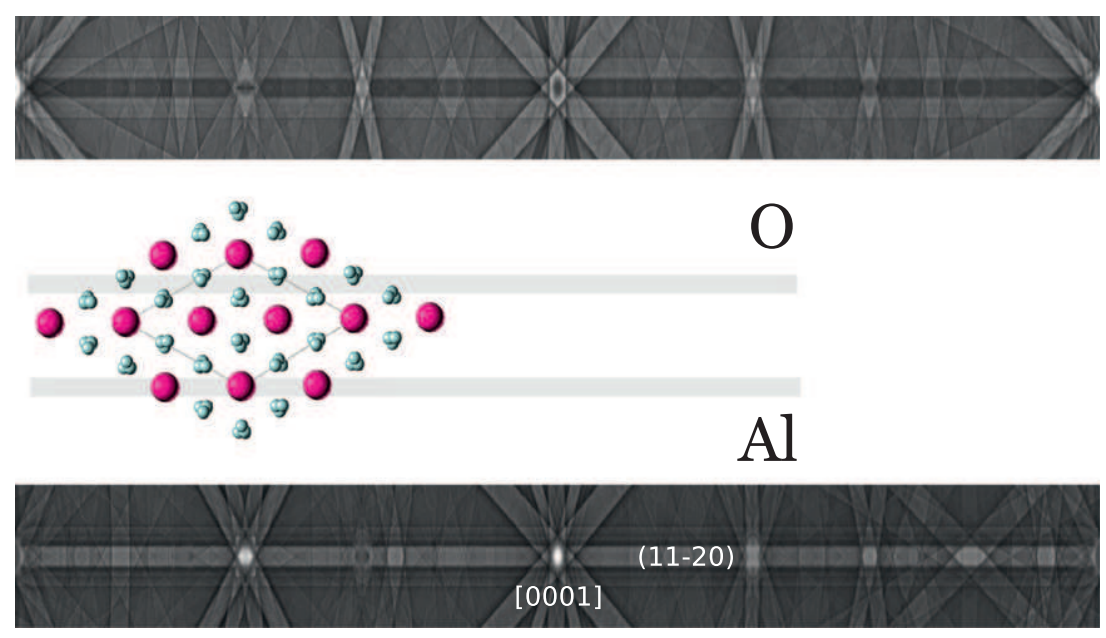

Fig. 2. Simulated element-specific Kikuchi patterns for the (1120) Kikuchi band in sapphire at an energy of $35 \mathrm{kV}$. In the middle, the crystal structure projected along the (0001) direction is shown with the respective atoms indicated. Top: pattern from 0 , bottom: pattern from $\mathrm{Al}$ (horizontal range: $\pm 90^{\circ}$ away from the [0001] direction,

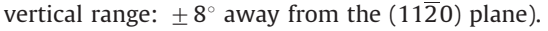

absence of diffraction effects for atoms directly on the sample surface. When the $\mathrm{Al}$ and $\mathrm{O}$ intensities are measured for directions corresponding to a line crossing a Kikuchi band, we obtain element-specific diffraction information, which can be connected to the crystallographic site of the $\mathrm{Al}$ and the $\mathrm{O}$ atoms in the sapphire crystal structure [19]. In order to illustrate this effect, in Fig. 2 we plot the simulated element-specific Kikuchi patterns corresponding to the experimental setup we have used [19]. One can nicely distinguish the inverted (1120) Kikuchi band contrast for the $\mathrm{O}$ atoms. The inverted contrast of the $\mathrm{O}$ band is due to the fact that the mean $\mathrm{O}$ position is physically exactly half way between the Al planes and thus, when the $\mathrm{O}$ atoms sample the minima of the Bloch wave field, the $\mathrm{Al}$ atoms sample the maxima (and vice versa) [19]. The working of this mechanism depends on the $\mathrm{Al}$ atoms dominating the dynamical diffraction effects (due to their higher atomic number), with the $\mathrm{O}$ atoms as smaller perturbations.

We see in Fig. 2 that a Kikuchi pattern is connected to the fixed specific localization of the respective source of backscattered electrons. This localization is provided by the quasi-elastic large-angle backscattering process. Large-angle quasi-elastic scattering also explains the remaining diffraction effects of inelastically scattered electrons with energies not too far from the primary beam energy [20]: inelastic scattering followed by a localized large-angle backscattering event in EBSD, will still produce a Kikuchi pattern [21]. In contrast, if (additional) inelastic scattering takes place after the backscattering event, then this produces new virtual sources which are not necessarily localized at the atomic positions. If the inelastic scattering involves delocalized valence band electrons (e.g. plasmons, electron-electron scattering), the incoherent sources will be distributed more evenly over the unit cell. The important implications of the delocalization of such incoherent events for the observed Kikuchi patterns are discussed in the next section.

\subsection{Incoherent event delocalization in the unit cell}

In the previous section, nuclear recoil at the atomic positions within the unit cell has been identified as a mechanism that provides the necessary fixed localization of incoherent scattering events for the formation of Kikuchi patterns. In this section we will investigate what happens if the incoherent scattering event is distributed evenly over the unit cell, as is expected to apply to e.g. the case of plasmon creation.
In order to illustrate the basic mechanisms we have chosen a model system of a simple cubic ( $s c$ ) crystal with an atom at $(0,0,0)$ in the unit cell (lattice constant $a=3.6 \AA$ ) and we calculate the Kikuchi patterns at $20 \mathrm{kV}$ including only the strongest bands. The patterns are displayed in gnomonic projection with the pattern center corresponding to the [001] direction. The horizontal and vertical extension of the patterns correspond to angles of $\tan (\theta)=1.5$ so that $\theta \approx 56.3^{\circ}$ with respect to [001]. The patterns simulate the expected variations of the signal due solely to diffraction and do not include e.g. the angular variation of the backscattering coefficient which is observed even for an amorphous sample. The exact values of any parameters we have used in the calculations have no influence on the mechanisms we will discuss here. In all plots shown here, a specific grey-scale value corresponds to the same intensity.

In Fig. 3 we show calculated Kikuchi patterns for electrons scattered from six different random positions within the unit cell of the same simple cubic model crystal. The difference between these patterns is due only to the different positions of the assumed incoherent events relative to the positions of the atoms which make up the crystal and which cause the diffraction of the emitted (EBSD) or the incident (ECP) electrons. From Fig. 3, we can see that the general underlying structure of the patterns (i.e. position and number of features) remains fixed while the intensities in the Kikuchi bands change with the position of the assumed event in the unit cell. It is clear that the intensity distribution of the Kikuchi pattern no longer has cubic symmetry, due to the usually lower site-symmetry of the assumed position of the incoherent event. This result can be seen as the generalized effect of the specific contrast inversion we have seen from the oxygen atoms in the lattice of sapphire discussed above. There, the relatively weakly scattering oxygen atoms sample some specific positions between the stronger scattering Al atoms.

It is currently not possible experimentally to position incoherent sources at arbitrary places inside a crystal unit cell, so that the individual Kikuchi patterns seen in Fig. 3 cannot be reproduced in an actual experiment. This is why we need to average all possible patterns from the potential positions of incoherent scattering in the unit cell if a scattering process contributes to an experimental pattern and does not differentiate between specific locations in the unit cell. This is shown in Fig. 4 for an increasing number of random positions. While the Kikuchi pattern is well defined for a specific single position, the average of 1000 different patterns from all over the unit cell displays a greatly suppressed diffraction 


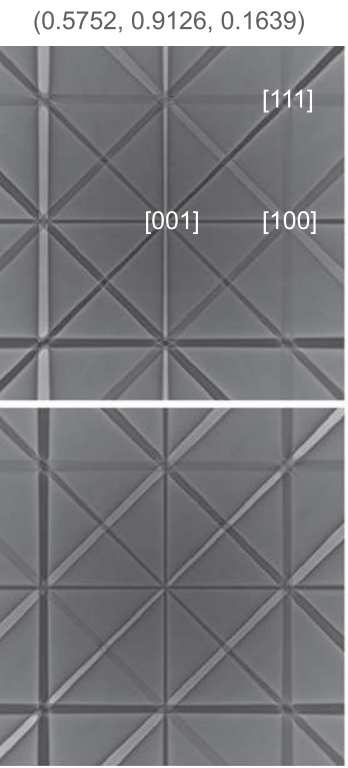

$(0.3186,0.6473,0.9455)$
$(0.8230,0.1575,0.4244)$

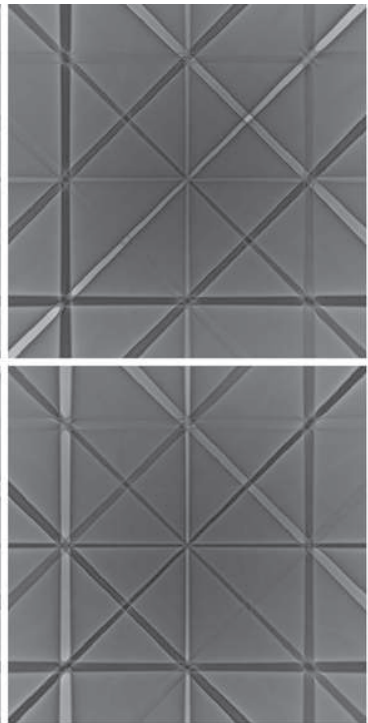

$(0.5664,0.8923,0.2061)$
$(0.0708,0.4024,0.6850)$

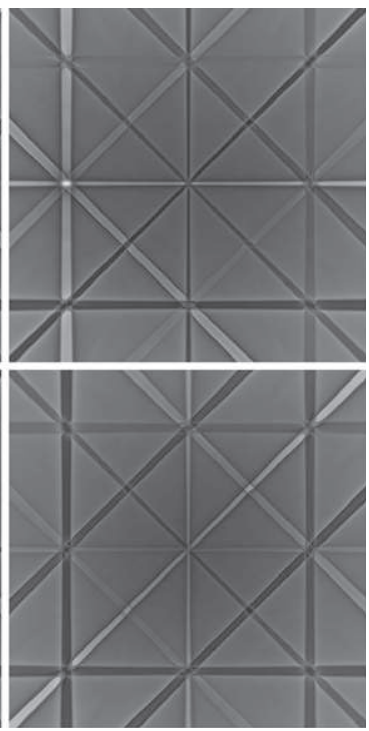

$(0.8142,0.1372,0.4666)$

Fig. 3. Individual Kikuchi patterns related to incoherent events occurring at six different random positions in a unit cell of a simple cubic lattice with an atom at $(0,0,0)$. The numbers give the $(x, y, z)$ location of the respective event in units of the lattice constant. Gnomonic projections, with pattern center at [001], horizontal and vertical angular extension up to $\tan (\theta)=1.5$. Low intensity is black, high intensity is white.
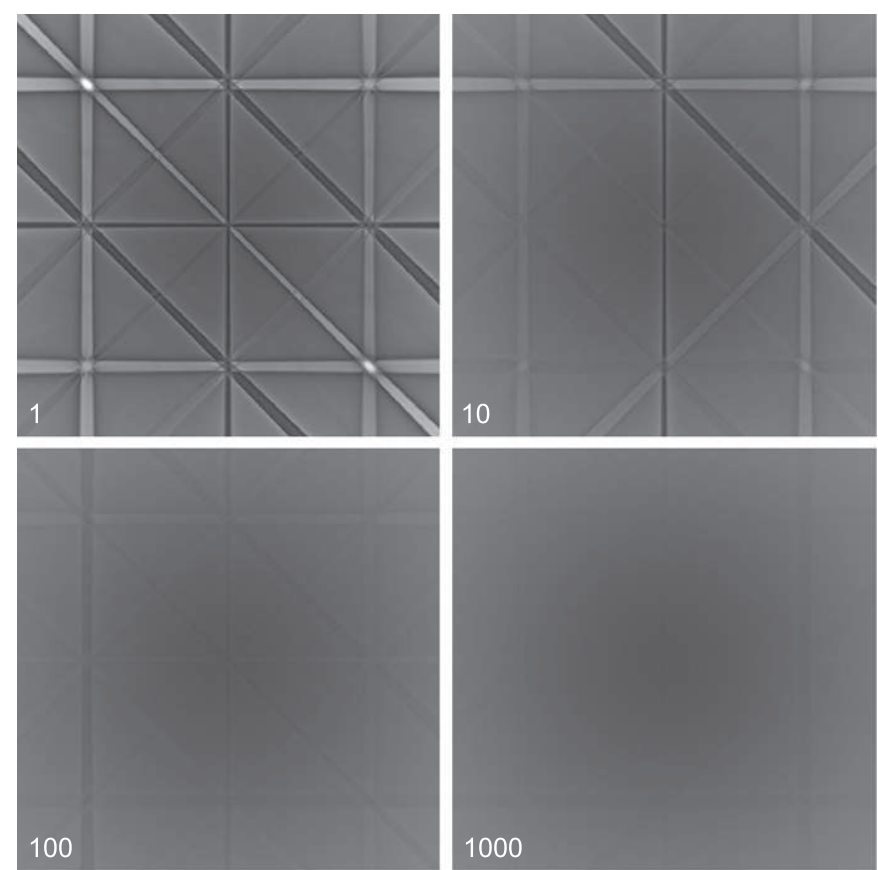

Fig. 4. Process of averaging out of the diffraction patterns by an increasing number of sources (as indicated) with random positions in the unit cell.

contrast (resulting in a featureless gray background). It is important to note that each of the different 1000 scattering processes, if repeated by itself from the same fixed position would produce a diffraction pattern that is characteristic of the respective site in the unit cell. In the scenario we considered in this section, the loss of diffraction information is not related to any imperfection of the crystal or spread of electron wavelength due to inelastic scattering, but it is purely due to the averaging of many "perfect" patterns from different source positions. In a simplified picture, each of these different Kikuchi patterns is produced one after another by subsequent electrons scattering inelastically at different locations (keeping in mind that a diffraction pattern actually cannot be measured for one single electron).

The gradual transition from a Kikuchi pattern of fixed, localized sources to a featureless background can be simulated by steadily increasing the random deviation of the source positions from the atom sites. This is shown in Fig. 5 for averaged patterns of a set of 100 incoherent sources for which the maximum allowed random deviation in the $x, y$, and $z$ coordinates from the atomic position increases from $0 \%$ to $100 \%$ of the lattice constant. We can nicely see the suppression of diffraction information with increasing deviation.

The averaging out of diffraction information by sources which are randomly distributed within a unit cell can be taken as a model for the description of the large group of background electrons in experimental EBSD and channeling patterns which do not seem to exhibit variations of the backscattering coefficient due to diffraction.

In particular, the effective averaging out of different Kikuchi patterns as described above can be caused by inelastic scattering. On the experimental side, measurements of the contrast in Kikuchi patterns as a function of the energy loss [20,21] have established the suppression of diffraction information by inelastic scattering. Specifically, plasmon creation is an important inelastic scattering mechanism which is not localized at the atomic positions. For silicon, a clear reduction in diffraction contrast was found if the outgoing trajectory is significantly longer than the inelastic mean free path [21], which in turn is mainly determined by plasmon excitation. Thus, in the case of EBSD, the trajectories of electrons with increasing energy loss will have an increased probability of containing plasmon excitation events on the outgoing path. These large-loss electrons will then mainly contribute to the featureless background. In contrast, the trajectories of low-loss electrons have a higher probability to contain a localized large-angle scattering as the last event, without any additional plasmon scattering afterwards (which is allowed to occur, however, before the large-angle scattering). Such trajectories then will give rise to a Kikuchi pattern. We have shown previously that detection of these "inelastic scattering before diffraction" trajectories is optimized by the scattering geometry 
0.0
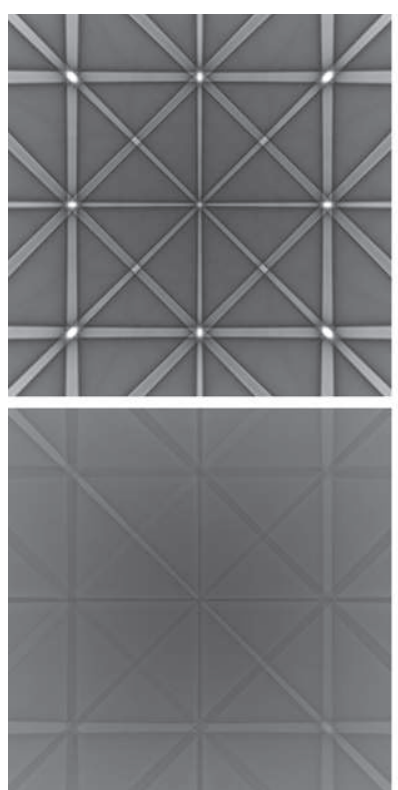

0.6
0.2
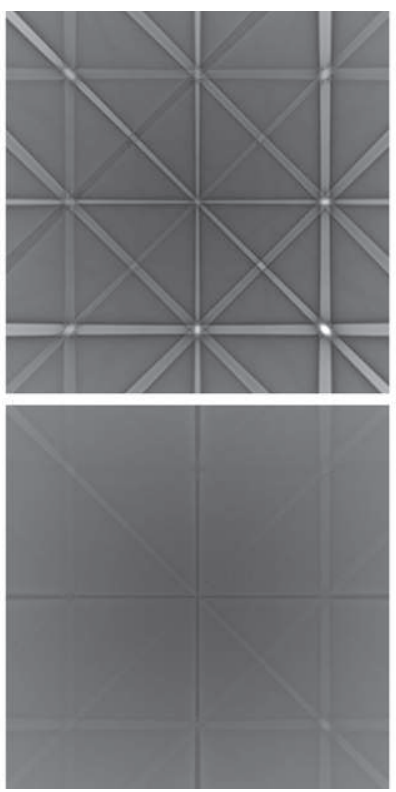

0.8
0.4
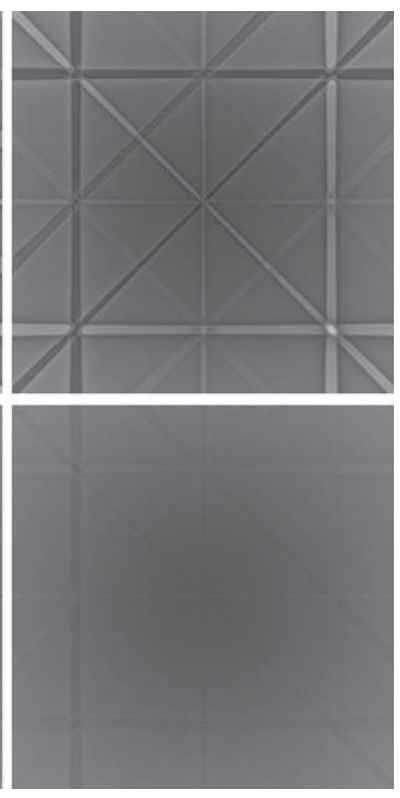

1.0

Fig. 5. Effect of increasing the allowed deviation of the Kikuchi sources from the atomic position. Averaged Kikuchi patterns for 100 sources at random positions in each case. The numbers indicate the maximum random deviation of the $x, y$, and $z$ coordinates of the sources from the atomic position in the unit cell (in units of the lattice parameter).

in EBSD with shallow incidence angles [21]. Our simulations thus explain the loss of diffraction information from the large-loss electrons in EBSD, as these are heavily influenced by plasmon excitation and other inelastic scattering. The remaining experimental intensity distribution without diffraction features should closely correspond to the angular variation of the backscattering coefficient in an amorphous material.

Compared to plasmon excitation, electron-electron scattering is expected to be rather homogeneously distributed for the case of scattering of $s p$ valence electrons but localized near the nuclei for scattering from $d$ electrons. Here we thus expect an intermediate behavior.

\section{Summary}

We have elucidated the central role of large-angle, quasielastic electron scattering and the connected nuclear recoil within the "channeling-in and channeling-out" model of electron backscatter diffraction (EBSD) and electron channeling patterns (ECP). The recoiling atom results in a localized incoherent electron source (in the case of EBSD) or detector (in the case of ECP) which is necessary to separate the coherent diffraction processes on the incoming and the outgoing part of the combined "channeling-in and channeling-out" process. Quasi-elastic Kikuchi pattern formation could thus be described as "channeling-in, recoil, and channeling-out". With the help of model calculations, we have shown that the localization of the incoherent scattering processes at specific fixed places within the unit cell is necessary to obtain a measurable Kikuchi diffraction pattern. We have extended the picture of "channeling-in and channeling-out" to incoherent sources located at general places within the crystal unit cell. This enables the treatment of the suppression of diffraction information from inelastically scattered electrons by averaging of different complementary Kikuchi patterns from various possible positions in the unit cell. As an outlook for the future, the ability to treat the "Kikuchi pattern electrons" and the "background electrons" in the same framework of dynamical electron diffraction theory might provide a way to combine diffraction with Monte-Carlo simulations of electron scattering. In this way, improved simulations of experimental EBSD and ECP patterns including any additional information in the pattern background might come into reach.

\section{References}

[1] A.J. Schwartz, M. Kumar, B.L. Adams, D.P. Field (Eds.), Electron Backscatter Diffraction in Materials Science, 2nd ed., Springer, Berlin, 2009.

[2] D. Dingley, Progressive steps in the development of electron backscatter diffraction and orientation imaging microscopy, Journal of Microscopy 213 (2004) 214-224

[3] D.C. Joy, D.E. Newbury, D.L. Davidson, Electron channeling patterns in the scanning electron microscope, Journal of Applied Physics 53 (1982) R81.

[4] A.J. Wilkinson, P.B. Hirsch, Electron diffraction based techniques in scanning electron microscopy of bulk materials, Micron 28 (1997) 279-308.

[5] G. Naresh-Kumar, B. Hourahine, P. Edwards, A. Day, A. Winkelmann, A. Wilkinson, P. Parbrook, G. England, C. Trager-Cowan, Rapid nondestructive analysis of threading dislocations in wurtzite materials using the scanning electron microscope, Physical Review Letters 108 (2012) 135503.

[6] Y.N. Picard, R. Kamaladasa, M. De Graef, N.T. Nuhfer, W.J. Mershon, T. Owens, L. Sedlacek, F. Lopour, Future prospects for defect and strain analysis in the SEM via electron channeling, Microscopy Today 20 (2012) 12-16.

[7] D.C. Joy, Channeling In and Channeling out: the origins of electron Backscattering and electron channeling contrast, Proceedings of EMSA (1994) 592-593.

[8] O.C. Wells, Comparison of different models for the generation of electron backscattering patterns in the scanning electron microscope, Scanning 21 (1999) 368-371.

[9] L. Reimer, Scanning Electron Microscopy_Physics of Image Formation and Microanalysis, Springer Verlag, Berlin, Heidelberg, New York, 1998. (ISBN 978-3-540-63976-3).

[10] S. Zaefferer, On the formation mechanisms, spatial resolution and intensity of backscatter Kikuchi patterns, Ultramicroscopy 107 (2007) 254-266.

[11] L.T. Chadderton, Diffraction and channeling, Journal of Applied Crystallography 3 (1970) 429-465.

[12] A. Winkelmann, C. Trager-Cowan, F. Sweeney, A.P. Day, P. Parbrook, Manybeam dynamical simulation of electron backscatter diffraction patterns, Ultramicroscopy 107 (2007) 414-421.

[13] A. Winkelmann, Dynamical Simulation of Electron Backscatter Diffraction Patterns, in: A.J. Schwartz, M. Kumar, B.L. Adams, D.P. Field (Eds.), Electron Backscatter Diffraction in Materials Science, Springer, Berlin, 2009, ISBN: 978-0-387-88136-2, 〈http://www.springer.com/cda/content/document/cda_ downloaddocument/9780387881355-c2.pdf?SGWID=0-0-45-749108-p173906909> (Chapter 2). 
[14] C. Maurice, K. Dzieciol, R. Fortunier, A method for accurate localisation of EBSD pattern centres, Ultramicroscopy 111 (2011) 140-148.

[15] M. De Graef, Introduction to Conventional Transmission Electron Microscopy, Cambridge University Press, Cambridge, 2003. (ISBN: 9780521620062).

[16] J.C.H. Spence, J.H. Zuo, Electron Microdiffraction, Plenum Press, New York and London, 1992.

[17] D. Van Dyck, Persistent misconceptions about incoherence in electron microscopy, Ultramicroscopy 111 (2011) 894-900.

[18] B.D. Forbes, A.J. D’Alfonso, S.D. Findlay, D. Van Dyck, J.M. Lebeau, S. Stemmer, L.J. Allen, Thermal diffuse scattering in transmission electron microscopy, Ultramicroscopy 111 (2011) 1670-1680.

[19] A. Winkelmann, M. Vos, Site-specific recoil diffraction of backscattered electrons in Crystals, Physical Review Letters 106 (2011) 085503.
[20] A. Deal, T. Hooghan, A. Eades, Energy-filtered electron backscatter diffraction, Ultramicroscopy 108 (2008) 116-125.

[21] A. Winkelmann, K. Aizel, M. Vos, Electron energy loss and diffraction of backscattered electrons from silicon, New Journal of Physics 12 (2010) 053001.

[22] M.R. Went, A. Winkelmann, M. Vos, Quantitative measurements of Kikuchi bands in diffraction patterns of backscattered electrons using an electrostatic analyzer, Ultramicroscopy 109 (2009) 1211-1216.

[23] J.M.F. Gunn, M. Warner, The effect of high momentum transfer on scattering from oscillators and crystals, Zeitschrift für Physik B Condensed Matter 56 (1984) 13-20. 